\title{
Swiss Medical Weekly: Guidelines for authors, 2001
}

The Swiss Medical Weekly accepts for consideration previously unpublished (even in lay journals) original articles from all fields of medicine. In addition to clinical topic manuscripts dealing with basic medical science are welcome if they are of 1) general and 2) clinical interest. For the submission of reviews we recommend authors to contact the editor's office at an early stage of manuscript preparation by e-mail [red@ smw.ch]. Prior to publication, all manuscripts, with the exception of Editorials and Letters to the Editors, undergo peer review. The Editorial Board may decline a paper on the basis of internal review and rapidly return the manuscript, usually within three weeks.

The guidelines for authors are based on the guidelines published by the International Committee of Medical Journal Editors (Uniform requirements for manuscripts submitted to biomedical journals. JAMA 1997;277:927-34).

http://jama.ama-assn.org/info/

auinst_req.html

\section{Categories of manuscripts:}

- Original clinical articles

- Original articles on clinical investigation and experimental medicine

- Basic science in medicine (by invitation only)

- Reviews (after prior consultation with the Editorial Board only)

- Minireviews (by invitation only)

- Editorials (by invitation only)

- Correspondence

Original articles can be submitted as "Short communications" not exceeding 1800 words or as "Rapid publications" (see below). The submission of case reports is discouraged. Novel observations including new laboratory findings or therapeutic approaches can be submitted as short communications.

\section{Submission of manuscripts}

Contributions must be written in English. The complete manuscript must be submitted to the publisher in electronic form as an email attachment or on a IBM formatted 31/4" disk (for address and technical details see below). As a rule, its length should not exceed 15 manuscript pages (about 2200 characters per page), including illustrations, tables, references, summary and key words. An accompanying written letter signed by all authors should follow by mail or facsimile. By their signature authors certify that they have read, checked and approved the manuscript. In addition to the title of the paper, the accompanying letter should also contain a statement to the effect that the manuscript contains original work and neither the whole nor any substantial parts of it (exception: abstracts) have been published in medical journals or the lay press, and that it is not being assessed by another journal with a view to publication. Publications partly duplicating material in the submitted paper should be enclosed with it. In the accompanying letter the authors should indicate any financial or personal relationships entered into in connection with the article submitted.

Where several authors are designated as responsible for an article, each individual should have made a substantial contribution at all stages of the article's preparation (conception and design, or analysis and interpretation of data; drafting of the article or critical revising; final approval of the version to be published). Participation solely in collection of data, performance of routine investigations, or general supervision of the research group does not justify authorship, nor does the fact of being in sole charge of the clinic or department in which the article was prepared; such contributions should be listed in the acknowledgements. In view of these requirements, more than six authors will be listed only in exceptional cases.

Outside financial support or other financial or personal relationships in connection with the submitted manuscript should be indicated in a footnote. Human and animal studies require approval by ethics committees. Where a study is in compliance with the standards of an ethics Committee, the ethics Committee concerned should be mentioned in the "Methods" section.

\section{Original papers}

(clinical or experimental)

Original papers should be organised according to the conventional pattern:

- Title page (date of draft, place of origin, hospital or institute; title; authors; exact address for correspondence including phone number and fax number and email address; details of financial support, national fund, etc.)

- Summary with 3-10 key words. The summary should inform the reader of the problem studied, the methods used, the principal results and the authors' conclusions. The summary should not exceed 250 words and should be divided into four sections: questions under study/principles, methods, results, and conclusions.

- Introduction with statement of the problems addressed

- Patients or observation material and methods (incl. statistical methods); where applicable, compliance with standards of ethics Committee; informed consent of the patients

- Results

- Discussion

- References

- Tables

- Figure legends

- Figures

\section{Short communications}

Short communications should not exceed 1800 words including abstract, key words, references, figure legends, and tables. The results and discussion section may be combined.

\section{Editorials}

Editorials should where possible not exceed six pages in length (no summary, but a list of not more than 10 references should be included).

\section{Reviews}

Review articles should be planned after consultation with the Editorial Board. We expect authors of Reviews to be experienced and acknowledged in the relevant field. Reviews should include a summary covering all essential points and a list of references.

\section{Letters to the Editor}

Letters should not exceed two pages (4500 characters), including illustrations, references, and tables.

\section{Rapid publication}

If authors feel that for reasons of general or public interest their manuscript requires more expeditious publication and quick processing through peer review, they may inform the Editorial Board by an e-mail inquiry indicating:

- The reasons the authors think the manuscript should be published immediately 
- The question(s) addressed by the study

- The results obtained

- The implications of the findings

Upon reception of such a request a member of the Editorial Board will contact the authors and negotiate the submission. SMW will review the manuscript within 4 weeks of submission and publish it within 6 weeks of acceptance of the final version.

\section{Preparation of manuscript}

Manuscripts should be prepared and submitted in electronic form using a standard word processor. Page numbering should be added throughout the manuscript, including figure pages. The date of submission of the manuscript and subsequent revisions should always be given on the first line of the title page.

The manuscript should be preferably submitted as an e-mail attachment with an attached letter of accompaniment or, where this is not possible, on a $31 / 4$ " or zip" disk. Customary abbreviations (e.g. ACTH, ECG) are permitted. All other abbreviations should be used sparingly and an alphabetic list of abbreviations with explanations should be supplied. Abbreviations that are used only once should be avoided. Always use SI units. Results in other units may be added in parentheses.

Tables and figures should be commented upon in the text and their position marked at the appropriate point. In the case of figures and tables from other publications, the source must be stated and copyright agree- ments provided. Lengthy tables should be avoided. Tables should be provided with captions, numbered consecutively and each reproduced on a separate page of the manuscript (not as a separate file!). Figures should each be submitted with legend on a separate manuscript page. Captions should be formulated to allow the figure to be understood without reference to the text. All abbreviations employed should be explained in the caption if not already included in the list of abbreviations. The figure should be imported using the jpeg format or tif (300 lpi) or eps format for drawings not exceeding a file size of $2 \mathrm{MB}$. With critical material we may request higher resolution illustrations during the review process. Upon acceptance of a manuscript we will negotiate with the authors the final submission of figures and photographs, either as high resolution files or glossy prints or slides. Photographs and colour illustrations are accepted at no extra page charge. If a figure or a table has been published, the original source should be acknowledged accompanied by written permission from the copyright holder for reproduction of the material.

\section{References}

The list of references should be arranged and numbered in order of appearance in the text, and not alphabetically. The appropriate number should be inserted in brackets [ ] in the text. The full titles of the quoted publications should be listed. List the first six authors followed by "et al.».
Examples:

1 Vega KJ, Pina I, Krevsky B. Heart transplantation is associated with an increased risk for pancreatobiliary disease. Ann Intern Med 1996;124:980-3.

2 Parkin DM, Clayton D, Black RJ, Masyer E, Friedl HP, Ivanov E, et al. Childhoodleukaemia in Europe after Chernobyl: 5-year follow-up. Br J Cancer 1996;73:1006-12.

3 Ringsven MK, Bond D. Gerontology and leadership skills for nurses. 2nd ed. Albany (NY): Delmar Publishers; 1996.

4 Philips SJ, Whisnant JP: Hypertension and stroke. In: Laragh $\mathrm{JH}$, Brenner BM, eds. Hypertension: pathophysiology, diagnosis, and management. 2nd ed. New York: Raven Press; 1995. p. 465-78.

All manuscripts should be sent in electronic form, and with a signed (by all authors) accompanying letter, to:

EMH Swiss Medical Publishers Ltd. SMW Editorial Secretariat

P.O. Box

CH-4010 Basel

Switzerland

Tel. $+41 / 61 / 4678552$

Fax +41/61/467 8556

e-mail:

for manuscripts: submission@smw.ch for letters to the editor: letters@smw.ch Editorial Board: red@smw.ch

Internet

http://www.smw.ch

http://www.emh.ch 